\title{
D'une école des sciences politiques
}

In: Politix. Vol. 1, №3-4. Été-automne 1988. pp. 6-12.

Citer ce document / Cite this document :

Damamme Dominique. D'une école des sciences politiques. In: Politix. Vol. 1, N³-4. Été-automne 1988. pp. 6-12.

doi : 10.3406/polix.1988.1346

http://www.persee.fr/web/revues/home/prescript/article/polix_0295-2319_1988_num_1_3_1346 


\section{D'UNE ECOLE DES SCIENCES POLITIQUES}

Créće en 1871, l'école libre des Sciences Politiques a immédiatement influé sur la configuration du champ universitaire et sur le fonctionnement du système politique et administratif. On s'interrogera ici sur les effets parallèles de l'institution de cette Ecole Politique et de cette Science Politique. Plus précisement, on tentera de ressaisir les usages sociaux et politiques d'une figure de la positivité scientifique et les fonctions sociales d'une école qui, longtemps, monopolisa l'apparence du monopole des sciences politiques.

Mais il n'est pas inutile sans doute de faire retour sur les origines de l'Ecole libre et de rappeler la conjoncture politique et intellectuclle dans laquelle elle est née ${ }^{1}$.

Emile Boutmy, son fondateur historiquement attitré, en présente le projet dans deux brochures de l'annćc 1871, Quelques idées sur la création d'une faculté libre d'enseignement supérieur (février 1871) ct Projet d'une faculté libre des Sciences Politiques (septembre 1871). Qu'y lit-on? Que l'institution constituc une ocuvre de salut national - elle s'adresse "aux esprits distingués qui ne se sont pas élevés au dessus du patriotisme et jusqu'à l'indifférence" 2 ; qu'clle apporte une solution à la crise d'autorité et de légitimité des élites politiques et sociales. Mais au travers et au-delà de la défaite militaire et de la crise du régime, l'instigatcur du projet reconnait dans les événements de 1870 et de 1871 les signes de la genèse de la société moderne, "l'avénement prochain et irrésistible de la démocratie dans le monde", le régne de la politique métaphysique qui contraint l'histoire à osciller entre révolution et dictature, l'urgence, enfin, d'une politique positive et d'une éducation scientifique, à l'exemple du voisin allemand. Pour lui, intellectucl, comme pour nombre de ses semblables, Sedan et Paris accomplissent l'annonce de Sadowa, réalisent la victoire de la science allemande. "C'est l'université de Berlin qui a triomphé à Sadowa ; on l'a dit avec une raison profonde ; et il faut être aveugle pour ne pas voir l'ignorance française derrière la folle déclaration de guerre qui nous a conduits où nous sommes (...); ne faut-il pas créer l'élite qui, de proche en proche, donnera le ton à la nation? Refaire une tête de peuple, tout nous ramène à cela" 3 .

"Faire une tête de peuple", c'est d'abord sauver "le culte du savoir et l'empire de l'esprit"4. De là, cette nécéssité d'une réforme du système universitaire et d'une propagation de l'esprit scientifique. C'est par la science positive que se fera la "réforme intellectuelle et morale" qu'exige l'heure.

D'un côté, en effet, le savoir constitue une ressource sociale, de l'autre la science positive détermine le politique.

Dans la socićté moderne, il n'y a de supériorité acceptée que celle enracinée dans le savoir, ct scul, il constitue un titre légitime de noblesse sociale. La domination politique - Boutmy parle d'hégémonie des classes qui se nomment elles-mêmes "les classes élevées" ne peut subsister et se reproduire qu'à la condition de s'appuyer sur le mérite. Ce mouvement méritocratique des sociétés démocratiques, Boutmy ne le déplore pas et l'acquisition d'une capacité intellectuelle lui parait le prix qu'ont à payer les classes supéricures pour maintenir leur position dans le système social ${ }^{5}$.

Mais le savoir n'offre pas sculement une source de compétence et d'autorité sociale ; il posséde également la proprićté de détruire ou de relativiser les théories abstraites, les "systèmes" dans la langue "savante" du temps. Micux que la religion et la morale, la science modeme peut corriger et stabiliser la démocratie parce que, en soi, elle contient une politique d'ordre. Entre Boutmy, Ernest Renan qui, au même moment note que le "rationnalisme est loin de porter à la démocratie"6 et Hippolyte Taine pour qui "la science engendre la prudence, et l'étude minutieuse diminue le nombre des révolutionnaires en diminuant le nombre des théoriciens"7, les correspondances sont évidentes; elles manifestent un moment, la crise "allemande" de la pensée française, et un segment du champ intellectuel du XIXème siècle.

\footnotetext{
1 Pierre Favre, "Boutmy et l'Ecole libre des Sciences Politiques", Revue Française de Sociologie, 1981, XXII, pp. 429-465, Dominique Damamme, "Genèse sociale d'une institution scolaire : l'Ecole libre des Sciences Politiques", Actes de la recherche en sciences sociales, 70, 1987, pp.31-46. Pour une synthèse sur la période, G. Weisz, The emergence of modern Universities in France , 1863-1914, Princeton, Princeton university press, 1980.

2 Emile Boutmy, Quelques idées sur la création d'une faculté libre d'enseignement supérieur. Paris, Lainé, 1871, p. 8.

${ }^{3}$ Emile Boutmy, art cit, pp. 4-5.

4 Id, pp. 5-6.

5 Sur la sélection sociale et scolaire au XIXéme siécle, cf. Ch. Charle, Les élites de la République, 1880-1900,

Paris, Fayard, 1987, notamment les chapitres I et II.

${ }^{6}$ E. Renan, La réforme intellectuelle et morale, Paris, Calmann-Lévy, s. d., p. 103.

7 H. Taine, "De la fondation d'une faculté libre des Sciences Politiques", Journal des débats, 17 Octobre 1871, in

L'Ecole libre des Sciences Politiques, 1871-1889, Paris, Chamerot, 1889.
} 
Dans la première brochure, Boutmy proposait une faculté d'enscignement supéricur, véritable encyclopédie des "Sciences d'Etat", solution plus propre, à ses yeux, à former une classe dirigeante compétente qu'une école spéciale d'administration. "Purement scientifique", ce plan déborde largement les frontières du politique, puisqu'il englobe la philosophie, les sciences et les arts, dessinant les lignes d'une "Ecole des Hautes Etudes des Civilisations Modernes", ou d'une section "Politique" de l'Ecole Pratique des Hautes Etudes, créée par Victor Duruy en 1869.

Or, cette université des sciences morales et politiques se révèle irréalisable en pleine Commune et, en toute hypothèse, décaléc par rapport aux attentes pratiques des groupes sociaux auxquels clle s'adresse. Dans le second appel, Projet d'une faculté libre des Sciences Politiques, de Septembre 1871, Boutmy travaille donc à accroître la visibilité de son école en resserrant son objet sur les seules sciences politiques. Cependant, pas plus sous cette forme que sous l'ancienne, l'institution ne peut être assimilée à une école professionnelle. Figure au centre de la visée l'instruction des élites politiques et administratives, mais le dispositif pédagogique ne se limite pas à un enseignement pratique en raison de la volonté d'inculquer un nouvel habitus à la classe dirigeante, de former une autre disposition à l'égard des groupes et des intérêts sociaux qui autorise l'objectivation nécessaire à "l'art social" dans un Etat moderne, c'est-à-dire à la "science du gouvernement" démocratique.

L'Ecole libre est inaugurée en Janvier 1872. Pauvre en hommes et en moyens, elle a alors pour tout local une salle louée à l'heure à la Société d'encouragement à l'Industrie Nationale, pour programme, cinq cours (Géographie et Ethnographic par Henri Gaidoz, Histoire diplomatique par Albert Sorel, Histoire des doctrines économiques par Anatole Dunoyer, Histoire des finances par Paul LeroyBeaulieu, Histoire des théories de réforme sociale par Paul Janet), pour public, un auditoire restreint (89 inscrits) composite et irrégulier ${ }^{1}$. L'Ecole, sous cette première forme, n'avait que de faibles chances de survic.

"L'Ecole n'est à aucun đegré une école professionnelle. Elle ne prépare pas à une carrière (alors que) dans toutes les institutions, on n'acquiert pas seulement du savoir, mais un moyen de vivre et une position dans le monde (...) Sous la forme actuclle, l'Ecole n'est pas viable (...) Nous avions la conviction que ces cours généraux, s'ils continuaient à n'être qu'un complément, une sorte de couronnement de l'ćducation libérale, sans répondre aux nécessités pratiques d'aucune carrière, réuniraient difficilement des auditoires nombreux et fidèles (...) Nous n'entendions pas renoncer aux visées supéricures en dehors desquelles notre ocuvre eût été pour nous sans intérêt et sans noblesse. Mais force nous était de prendre un second point d'appui sur un sentiment plus stable et plus général que la curiosité scientifique" 2 . Pour "faire vivre l'affaire", il fallait attacher l'"ocuvre" aux sciences camérales (Cameral-wissenschaften, ou science du gouvernement, des universités d'Outre-Rhin). En Juillet 1872 , la décision est prise de créer deux sections, diplomatique et administrative. Au bout d'un an, le premier intérêt de curiosité étant épuisé, nous nous sommes trouvés en présence d'auditoires diminués, et, ce qui est pis, absolument instables (...) L'Ecole périssait. (...) Nous avons institué un enseignement formé, pour partie, d'un véritable apprentissage professionnel. L'effet a répondu à notre attente. L'Ecole a reconquis rapidement la faveur publique"3.

C'est alors que véritablement s'opère le travail d'emboîtement entre les premiers appels, pensés dans une logique intellectuelle et politique et un dispositif professionnalisé, inćvitable pour une fondation privéc mais accordé également à l'esprit d'entreprise de Boutmy, à son désir de jouer un coup et de le gagner et appuyé par les milicux d'affaires, banquicrs, dirigeants de sociétés, hauts-fonctionnaires, hommes politiques, gagnés à sa cause, qui par là même, devenait leur. Car le don appelle le contre don, et les souscripteurs, "hommes pratiques", socialement intéressés, avaient entendu contribuer au développement d'une école spéciale d'administration.

Ainsi, là où l'on tendrait à voir un acte inaugural d'institution, perçu et pensé rétrospectivement comme tel alors même que Boutmy lui-même parle de "programme hâtivement rédigé", ou de sa recherche "pour assurer pendant trois ans (...) la marche d'une ocuvre qui n'était alors qu'une idée dans une tête humaine - une "chimère" (Rapport au conseil d'administration, 1895) -, l'analyse découvre une suite d'ajustements entre une offre culturelle et des demandes sociales. L'Ecole libre des Sciences Politiques s'est faite à travers une succession de décisions, réactions ou anticipations, de mieux en mieux accordées au champ social et au marché universitaire.

La question de la constitution d'une science politique, ou encore du rapport entre science et politique que noue l'Ecole, implique, à notre sens, le double détour d'une anlyse de la caméralisation des sciences politiques (i.e. de leur transformation en sciences du gouvernement) et d'une étude du scientisme, de ses modes et de ses usages.

\footnotetext{
${ }^{1}$ Sur les commencements de l'école, cf Damamme, art. cit.

2 E, Boutmy, ELS.P Assemblée générale des actionnaires, Paris, Martinet, 1872, pp. 13-14.

${ }^{3}$ E, Boutmy, Lettre au Ministre de l'Instruction Publique, s. d., Archives d'Histoire Contemporaine.
} 
Faut-il souligner d'abord que la logique de la situation aboutit à la domination des disciplines juridiques, notamment des sciences administratives. De même, doit-on s'étonner que, savoir de l'Etat et savoir pour l'Etat, l'Ecole s'ouvre à ce vaste espace d'objets, à la fois consacrés et controversés, que forment les questions sociales. Si "les plus retentissantes(...) ne se prêtent pas à un enseignement ou à une réfutation scientifique, parce qu'clles empruntent tout au rêve, rien aux faits et à l'expérience (...), il est d'autres questions sociales d'un ordre plus positif, par exemple la réforme de l'impôt et surtout ce qu'on appelle les questions ouvrières. Il ne s'agit pas ici de reconstruire la société sur un plan nouveau"1.

En deçà de ces marques, les plus visibles, on perçoit que la caméralisation touche l'ensemble des enscignements et que, posant l'action à l'horizon de tout savoir, la pratique en ligne de fuite de toute connaissance, le processus opère l'instrumentalisation de la totalité des savoirs. Davantage : leur institutionnalisation s'accomplit sclon cette même perspective ordonnée par le point de vue du pouvoir et de la décision. Les contraintes des marchés, universitaire, politique et administratif, contribuent ainsi à façonner l'orientation des sciences politiques, leur positvité, comme elles apparaissent également au principe de l'appropriation et de la reconnaissance de sciences sociales encore marginales dans l'université, tclles l'économic, la statistique, la géographic économique, l'ethnographic ou l'histoire politique contemporaine, sans nul doute, l'"invention" propre de l'Ecole.

"Vous savez le rôle de plus en plus considérable que jouent les questions économiques dans la vie moderne (....) Non sculement l'étude du budget français mais aussi celle des budgets étrangers est une excellente préparation. Mais beaucoup d'entre vous se destinent aux Affaires étrangères; or permettezmoi de vous dire que la diplomatic plus que jamais s'appuic sur des questions financières. Le comte Goluchowsky le disait très justement: "les guerres commerciales sont les guerres de l'avenir. N'oublic\%. pas, mon cher James, que la moitié des actions humaines est encore dictée par le sentiment. Tout n'est pas intérêt en ce monde mais il ne faut pas nier le facteur économique et, après tout, il n'est pas aussi moderne que cela" 2 .

Si le projet caméral, enfermant les savoirs dans la logique pratique de l'art social, les inscrivant dans une perspective fonctionnelle, produit des sciences de la décision politique, on peut faire l'hypothèse, toujours au titre des conditions de possibilité et d'ćnonciation des savoirs, que leur présence dans une institution scolaire, autorise une conception fédéraliste, formellement égalitaire des connaissances, et favorise l'extension du domaine plus que son organisation, car, à partir du moment où la recherche d'efficacité et de rentabilité devient prépondérante, s'impose l'obligation de quadriller systématiquement l'ensemble du territoire reconnu, mais aussi, potenticllement, de l'accroître sans fin. Nécesssité faite vertu, la définition du champ des connaissances politiques, compris comme accumulation éclectique de savoirs spéciaux, avec, d'un côté, des sciences institutionnalisécs dans leur forme d'existence canonique, académique et universitaire, "déjà-là", résultats ossifiés de luttes de classification au sein du champ intellectucl, et, de l'autre, des domaines empiriques à délimiter et à rationaliser, découle ainsi de la ligne d'action retenue. Logique pratique et formes de positivité que n'épargnera pas, comme on sait, la critique des sciences politiques d'Emile Durkheim ${ }^{3}$.

"Le propre des sciences d'Etat est qu'elles ne forment pas un ordre défini de sciences; elles sont plutôt dans leur genre une encyclopédic (...). Les méthodes de ces sciences ne les divisent pas moins que leurs sujets. Les unes sont purement descriptives, d'autres inductives, d'autres déductives ; les unes classent les faits, d'autres dégagent des lois, d'autres tirent les conséquences d'axiomes préétablis (...) Le groupe historique ne doit pas être seulement l'une des parties de l'enseignement des sciences d'Etat ; il doit en être la partic prépondérante. Il convient que les autres groupes lui empruntent, non pas précisément sa méthode, mais son point de vue et son cadre. Au sujet de la section des ćtudes administratives, je ferai une seule recommandation. La considération assidue de systèmes aussi compliqués que nos différents services administratifs (...), engendre cette fâcheuse habitude d'esprit qui consiste à ne plus pouvoir les imaginer que comme ils sont et chacun à sa place ordinaire(...), et à envisager enfin le plus petit dérangement comme un trouble grave qui se répercuterait profondément dans toute la masse (...). Le groupe des sciences ćconomiques a aussi sa faiblesse propre et son danger. Sans cause que je sache saisir, cet ordre d'études est resté singulièrement scolastique et abstrait" ${ }^{4}$.

S'interrogeant sur le coût scientifique des légitimations sociales et savantes, Boutmy estimait pourtant qu'il n'avait pas sacrifié "la pensée première qui inspirait les fondateurs". On ne s'est pas fait faute de lui opposer le bilan comptable des programmes, la mise en place d'une école d'administration, moins le

\footnotetext{
${ }^{1}$ Les sciences sociales et les questions sociales, m. s., A. H. C repris in "Observations sur le projet présenté au conseil général des Facultés", 1893, A. H. C.

2 P. Leroy-Bcaulicu, Cours de science financière, 1903, A. H. C.

${ }^{3}$ E. Durkhcim, La science sociale et l'action, Paris, P. U. F, 1970. Leçons de sociologie, Paris, P. U. F, 1969.

${ }^{4}$ E. Boutmy, "Observations sur l'enseignement des sciences politiques et administratives", Revue internationale de l'enseignement, 1881, I, pp. 237-249.
} 
nom. Reste qu'à se satisfaire de ce jugement, on gommerait complétement les limites de la professionnalisation ; on manquerait aussi le sens politique de l'entreprise comme les instruments utilisés pour l'atteindre. L'Ecole pouvait-elle se contenter d'un enseignement pratique sans se rabaisser et se perdre symboliquement et matériellement en abandonnant la force de légitimation que lui fournissaient la science et la croyance dans la science, et surtout sans amputer sa visće indissolublement politique et scientifique?

Boutmy aimait à répéter que l'Ecole appartenait au parti de la science. Or, prendre position pour la science, c'est prendre aussi parti pour une politique. La science de l'Ecole conforte en effet l'idéologic libérale et, bonne fille, justifie toutes les politiques, "opportunistes", prudemment "progressistes", ou conservatrices qu'on pouvait souhaiter ; conditionne, d'abord, l'accord entre "science" et politique une définition formellement empiriste et positiviste du savoir : les faits, rien que des faits, toujours plus de faits. Au-delà, cette harmonic, d'apparence spontanće, résulte de deux mécanismes principaux. A un effet de théorie, ou effet-méta, s'ajoute un effet-méthode, qu'il soit de nature historique ou expérimentale. Pouvait-on, sur ce point, être plus explicite que le maître et l'ami d'Emile Boutmy, H. Taine: "Dans une science, la méthode est tout, elle en fait l'esprit, elle en fixe d'avance les conclusions ; la nôtre est anti-jacobine"l. Pour Boutmy, les sciences politiques doivent d'abord faire comprendre que "toutes les questions sont difficiles, et la plupart des solutions complexes" 2 . Derrière cette formule se profile l'effet-théorie : la "complexité" des "phénomènes" impose qu'on les décompose en éléments simples, qu'on fractionne l'objet, qu'on le sérialise. Procédure en quelque sorte homologue à la politique "scientifique" des libćraux et des opportunistes - "Croyez, déclarait Gambetta, qu'il n'y a pas de remède social, parce qu'il n'y a pas une question socialc. Il y a une série de problèmes à résoudre." (Louis Gambetta, Le Havre, 18 Avril 1872). Outre que cette complexité trace une séparation entre doctes et ignorants, qu'elle contribue, par cette mutation, à fonder le pouvoir des experts, elle rend problématique toute décision. Sans travestir la formule de Boutmy, on concluera que la "scientifisation" des "questions" porte, sinon à l'abstention, du moins à la prudence. La compétence se résout en une morale de sagesse : on sait de quoi l'enfer est pavé, et que les intentions les plus pures emportent les conséquences les plus perverses. Appréhender le récl dans son épaisseur, se défier des systèmes, par essence "a priori", dévoiler et mesurer "les fléaux du bien", ces stratégies du savoir -sociologique des effets émergents avant la lettre -, s'analysent comme les principes d'une science pratique de l'action et, en deçà, et plus fondamentalement, comme un stock de dispositions à inculquer.

"Ainsi, la théorie de l'impôt progressif n'est pas rationnelle ; elle ne sort pas d'une analyse exacte des faits sociaux ; elle est superficiclle ; ce n'est pas une doctrine scientifique. Cette théorie est en outre dangereuse, parce que partant du principe de l'égalité de sacrifice, elle a une tendance invincible à vouloir corriger les inćgalités sociales; il y a là un entraînement qui peut être fatal" 3 .

L'important n'est pas dans la conclusion de Leroy-Beaulieu ni même dans l'information qu'clle soustend, mais dans le type de démonstration, dans le travail du négatif, qui transforme une proposition en une erreur scientifique, et qui, en changeant le terrain du débat, la fait passer de la doxa à l'épistémè. Car que répondre à une démonstration scientifique?

Tout aussi essentielles apparaissent l'interprétation et l'utilisation de la connaissance expérimentale et de la critique historique. A travers les cours d'histoire, de géographic, d'cthnologic et d'économic, l'Ecole sc souvient de la théorie des sciences de la culture d'H. Taine. C'est dans la préface à l'"Histoire de la littérature anglaise" (1864) que le philosophe a développé dans toutes ses conséquences sa problématique de l'histoire des civilisations, conçues comme des totalités dont l'unité et la stabilité ressortiraient d'une faculté maîtresse, effet elle-même de l'interaction de trois déterminants, la race, le milicu, le moment. Texte fondateur pour Boutmy puisqu'il s'agissait de la découverte d'un nouveau continent scientifique, la science de la formation et de l'évolution des systèmes sociaux et politiques. "Ce qu'Aristote a fait pour le raisonnement dans ses analytiques, $M$ Taine, dans la préface de la Littérature Anglaise, vient de le tenter et de l'accomplir ; l'histoire est maintenant en possession de sa méthode $4 "$. L'histoire est une science, elle est la science. Bref, de Taine, Boutmy va hériter d'une théorie des systèmes sociaux et, méléc aux influences de l'empirisme et du positivisme, d'une méthode d'analyse.

"Le groupe historique, indiquait-il, ne doit pas être seulement l'une des parties de l'enseignement des sciences d'Etat ; il doit en être la partie prépondérante. Il convient que les autres groupes lui empruntent (...) son point de vue et son cadre (...). L'histoire a ce merveillcux privilège qu'elle vit d'examen, qu'clle est une école de modération et de patience (...), qu'clle fonde le culte partial de la patrie sur la tradition nationale" 5 . Mais l'histoire n'est pas seulement mémoire de la patrie, savoir à la recherche de régularités

${ }^{1} \mathrm{H}$. Taine, Lettre manuscrite à E. Boutmy, 5 Juillet 1881, Archives Boutmy.

2 E. Boutmy, Projet d'une faculté libre des sciences politiques, Paris, Lainé, 1871, p. 9.

3 P. Leroy-Beaulieu, Traité de la science des finances, Paris, Guillaumin, 1887, Vol II, pp. 137 et sq.

${ }^{4}$ E. Boutmy, "M. Taine et la nouvelle méthode historique", La Presse, ler Juin 1864 et sq.

5 E. Boutmy, "Observations...", art cit. 
et de nécessités ${ }^{1}$, ou encore modalité discursive, "point de vue" ou "cadre" dans lequel viennent s'ordonner les énoncés les plus divers, le droit comme l'économie. Elle est tout cela à la fois et c'est elle qui apprend que le temps tisse des fils indestructibles : le "temps et le travail créent seuls les institutions durables, rien de solide ne s'élève sur table rase, sans racine dans le passé" 2 . Du passé, impossible de faire table rase ; il est des permanences, des invariants contre lesquels la volonté humaine ne peut rien, contre lesquels l'action se révèle dangereuse quand elle s'avise de les négliger ou de les contraindre. Si tout le réel n'est pas rationnel, ce n'est pas sans danger qu'on cherche à forcer la réalité à ressembler à l'idéal. L'histoire, par son réalisme et par son déterminisme, emporte dévoilement des mythes et des idćologies, qu'elles soient jacobines, socialistes, radicales ou rétrogrades; elle enferme une critique qui se veut décisive de toute théorie volontariste de l'action politique; elle enseigne en même temps la force des choses et leur relativité, et, en cela, elle apparait au centre d'un projet de modernisation conservatrice, écho libéral de l'Appel aux Conservateurs d'Auguste Comte pour un progrès ordonné, c'est-à-dire pour l'ordre comme condition du progrès.

Mais le rapport de détermination entre science et politique n'est pas moins immédiat dans le cas des sciences expérimentales, notamment au travers de l'économie. Selon Boutmy, "la règle à suivre dans l'organisation de cet ordre d'enseignement est d'y faire prévaloir l'observation par les enquêtes, d'y introduire amplement le procédé statistique et, d'autre part, de ne pas le considérer comme formant à lui tout seul un monde qui se suffit, de ne pas attribuer une valeur absolue et impérative à des formules où les éléments moraux de la société ne figurent pas à côté des éléments économiques (...) Les sciences économiques, surtout dans leur phase actuelle, forment un contrepoids très nécessaire à une influence abusive qu'exercent en commun les sciences historiques et les sciences administratives. Ces deux derniers ordres d'études ont un péril propre qui est d'éxagérer l'idée de l'Etat"3. Au delà de la signification politique de la science ćconomique, ces dangers de l'idée d'Etat complaisamment dénoncés par Boutmy, histoire et statistique libèrent le raisonnement économique des risques de divagation de l'analyse déductive. En résumé, si l'économie est vraiment ce savoir scientifique qu'elle prétend être, clle doit s'abstenir de détruirc des lois de principes a priori et se limiter à énoncer des propositions induites de faits ou de séries de faits, mathématiquement controlés, vérifiés sur le long terme ou confrontés à des expériences antérieures ou étrangères. Pourtant respectueuse des "faits" depuis J. B. Say, l'orthodoxic libérale paraissait encore trop imprégnée d'abstraction aux économistes de l'Ecole.

"Pour assurer sa marche, sans se laisser troubler par les bruits que soulèvent les faux systèmes, l'économie politique dispose d'une méthode qui a renouvelé toutes les autres sciences, et qui s'applique avec non moins de fécondité aux questions économiques et sociales : celle de l'observation"4. Ou, après ce permier témoignage d'Emile Cheysson, cet autre texte extrêmement révélateur : "l'économie politique est une science quand elle observe les faits, les décrit et en cherche les lois (...). Tout autre est la position du socialisme sur le terrain économique. Le socialisme n'est pas, comme l'économie politique, une science, car il ne se propose pas comme objet d'observer les phénomènes pour en induire des lois. Les observations qu'il recueille, il les produit surtout à l'appui de la critique qu'il fait de la science et de la condamnation qu'il prononce contre la société"5.

Conséquence logique de cette critique de l'abstraction de l'économie et de sa prétention à s'ériger en théorie générale du système social, le recrutement de praticiens et d'universitaires formés à la statistique d'un côté, A. de Foville, E. Cheysson et R. Stourm, de l'autre, E. Levasseur et P. LeroyBcaulieu, et d'économistes partisans d'un recours à l'analyse historique, A. Pigeonnau mais surtout E. Levasseur.

Emile Cheysson, 1836-1910.

Ecole Polytechnique ; ingénieur des Ponts et Chaussées ; secrétaire de Le Play à l"Exposition Universelle de 1867. Directeur des usines du Creusot, 1871-1874; Directeur des cartes et plans au Ministère des Travaux Publics, 1874-1884.

Membre de la société d'Economic sociale.

Professeur d'économie industriclle à l'Ecole des Mines, 1885-1901; professeur d'économie à l'Ecole de 1882 à 1889 et d'ćconomie sociale à partir de 1891. E. Cheysson fait le lien entre les libéraux et les leplaysiens.

1 A la fin de l'introduction, d'inspiration très tocquevillienne, de "L'Europe et la Révolution Française", A. Sorel conclut "jestimerais que mon travail n'a pas été inutile, si je parvenais à ce résultat : montrer dans la Révolution Française (...) la suite naturelle et nécessaire de l'histoire de l'Europe, et faire voir que cette révolution n'a point porté de conséquence, même la plus singulière, qui ne découle de cette histoire et ne s'explique par les précédents de l'ancien régime." A. Sorel, L'Europe et la Révolution Française, Paris, Plor, 1892, vol. I, p. 6 et 7.

2 R. Stourme, Les finances de l'Ancien Régime et de la Révolution, Paris, Guillaumin, 1895 Vol II, p. 502.

3 E. Cheysson, "De l'économie politique", in Oeuvres choisies, Paris, Rousseau, 1911, vol. I.

4 E. Levasseur, Questions ouvrières et industrielles en France sous la IIle République, Paris, Rousseau, 1907, p. 417.

5 E. Cheysson, op cit. 
Président de la commission centrale de Géographie, de la société de géographie commerciale, de la société de statistique, de la société de médecine publique, de la ligue nationale contre l'alcoolisme, de la sociétć générale des prisons, de l'Union centrale du patronage des libérés, de la société des habitations à bon marché, de l'association des assurances sociales. Vice-président du Muséc social, de la ligue nationale de la mutualité, de l'Alliance d'hygiène sociale...

Académie des Sciences Morales et Politiques, 1901.

Alfred de Foville, 1842-1913.

Ecole polytechnique et droit ; auditeur au Conseil d'Etat, 1866 ; Sous-chef de cabinet de PouyerQuertier, 1871-1872, sous-chef de bureau au Ministère des Finances, 1872-1876 ; chef adjoint du cabinct de Léon Say, nommé par L. Say, chef du bureau des statistiques, 1877-1893 ; Directeur des Monnaies et Médailles, 1893-1900; Consciller Maître à la Cour des Comptes, 1900.

Lauréat de l'Académie des Sciences Morales pour La variation des prix ; éditeur de F. Bastia ; collaboratcur à $L ' E$ conomiste Français .

Professeur d'Economic à l'Ecole de 1879 à 1883 , de 1889 à 1893 puis, de nouveau à partir de 1899 ; professeur à l'Ecole des Arts et Mćticrs, 1882-1893.

Président de la Société de statistique de Paris ; Membre de l'Académie des Sciences Morales, 1896, secrétaire perpétuel, 1909.

Paul Leroy-Beaulieu, 1843-1917.

Fils d'un préfet de la Monarchie de Juillet et député du Calvados de 1848 à 1859, de la clientèle de Guizot.

Etudes à Paris puis à Rome, Bonn et Berlin.

Couronné quatre fois par l'Académie des Sciences Morales et Politiques: De l'influence de l'état moral et intellectuel des populations ouvrières sur le taux des salaires (1867), De la colonisation chez les peuples modernes (1870), De l'Administration locale en France et à l'étranger (1870), De l'impôt foncier et de ses conséquences (1870).

Rédacteur au Journal des Débats, collaborateur de la Revue des Deux-Mondes, fondateur de L'Economiste Français (1873).

Suppléant de son beau-père, Michel Chevalier, à la chaire d'économie du Collège de France, puis titulaire de la chaire en 1878.

Professeur de science financière à l'Ecole de 1872 à 1880.

Membre de l'Académic des Sciences Morales et Politiques, 1878

Emile Levasseur, 1828-1911.

Ecole normale supéricure ; agrégé d'Histoire, 1854 ; professcur au Lycée Napolćon, 1854-1868.

Trois fois lauréat de l'Académie des Sciences Morales et Politiques, La question de l'or (1858), Histoire des classes ouvrières en France depuis la conquête de Jules César jusqu'à la Révolution (1859), IIistoire des classes ouvrières depuis la Révolution (1867).

Professeur de géographie, histoire et statistique au Collège de France de 1872 à 1911. Professeur de statistique et de géographie économique à l'Ecole de 1872 à 1911, et au Conservatoire des Arts et Métiers de 1876 à 1911.

Membre de l'Académic des Sciences Morales et Politiques, 1868.

Président de la société d'économie politique, de la Socićté de statistiques de Paris, vice-président de l'Institut international de statistiques, membre de la Société de géographie, de la Société de nationale d'agriculture...

Si donc l'Ecole ne s'écarte pas de l'orthodoxie libérale, l'économie voit rejeter catégoriquement sa prétention à fonder, et par là-même à expliquer, l'organisation sociale - elle ne constitue ni l'infrastructure de la société, ni le ressort en dernière instance de son évolution. Les formes économiques ne peuvent être dissociées des sociétés et de leur évolution. L'introduction de l'histoire, par la dénaturalisation des phénomènes "économiques" qu'elle opère, relativise les lois qu'énonce la science orthodoxe, et parallèlement le recours massif à la statistique, en diminuant le caractère abstrait de la discipline, accentue son rôle d'outil pour l'action.

Pour résumer, l'histoire, comme la statistique, conferrent un supplément de "réalité", et donc d'autorité, aux démonstrations économiques. En même temps, devant des faits plus déterminés, plus précis mais aussi plus plastiques, l'économic gagne en force performative : elle dit davantage du monde pour agir davantage sur le monde.

L'invention de "Science Po", école d'administration et école de politique, rend donc visible un ensemble épars et mouvant de disciplines, qu'elle baptise sciences politiques, hybride de sciences sociales et de sciences de gouvernement, les unes tournées vers l'apprentissage de règles et de techniques, les autres attachées à la découverte de lois. Sans même évoquer ici l'étude des institutions politiques el administratives, au développement de laquelle elle apporta une contribution majeure, ou encore l'accumulation de savoirs et de technologies impliqués par la croissance des sciences gouvernementales, on observera que la théorie des systèmes sociaux propre à l'Ecole ne fut pas sans effet de connaissance, car de l'analyse des races et des milicux, des institutions et des symbolisations qui trament "une civilisation", cheminent des voies diverses de recherches qui aboutissent à Mosei Ostrogorski, Elie 
Halévy et à André Siegfried. Pourtant ces travaux qui demeurent, et dont la présence durable aveugle notre regard contemporain, ne forment alors qu'une fine pointe de ce continent sans frontière que constituent alors les sciences politiques : des sciences au service du politique pour une politique au service de la science.

Ce statut, et cette finalité, jl est clair qu'ils ne furent pas sans conséquence. Dans l'ordre d'abord des représentations politiques. Peut-être est-on maintenant micux en mesure de comprendre ce que Boutmy laissait entendre lorsqu'il affirmait son adhésion au scul parti de la science : la science objective, elle médiatise. L'apprentissage des sciences politiques commande la formation des hommes d'Etat dans les régimes démocratiques, les Républiques, parce que la science autorise potentiellement une distanciation par rapport à son parti, à son intérêt, à son opinion, en d'autres termes, elle tend à libérer de la particularité. Bref, si l'Etat moderne implique du savoir, le savoir conditionne l'accès au gouvernement de l'Etat.

Représentations politiques, mais aussi représentations sociales. Insćrant les sciences politiques dans la perspective de l'action et de la décision, l'Ecole les ordonne à partir de la position de l'homme d'Etat, travaille ainsi à façonner la représentation institućc du politique, faisant du pouvoir, de ses institutions, de ses intérêts permanents et de ses compétences légitimes (ou illégitimes) un objet d'étude systématique. C'est souligner combien elle a contribué, tant sur le mode pratique que sur le mode théorique, non seulement à l'imposition d'une science politique (toujours au sens de politique scientifique) mais aussi à l'objectivation de l'Etat.

Le processus, en outre, s'avère d'unc importance décisive dans l'histoire de la construction du système administratif. A la fois pratiquement et symboliquement. Car, organiser une formation scientifique des fonctionnaires, c'est en effet reconnaître la nécessité de la compétence, autrement dit, rendre sensible cette nécessité et manifester cette compétence. L'Ecole montre que pour administrer un Etat moderne, pour être un haut-fonctionnaire, il faut assimiler une masse de connaissances spéciales, et que la compétence générale des élites politiques se constitue précisément à partir de cette pluralité de savoirs particuliers. Ainsi, l'Ecole, par sa présence comme par ses exigences, participe-t-elle activement à la fabrication et à l'autonomic de la haute fonction publique : d'un côté, clle rationalise le système de préparation aux concours, marché atomisé de petits producteurs indépendants, de l'autre, elle autorise un travail pédagogique systématique, social autant que technique, au moment où clle déplace, partiellement, les limites de la sphère sociale du recrutement au delà des héritiers directs des anciennes classes dirigeantes, modifiant les mécanismes de l'héréditć professionnelle et de la reproduction sociale. Plus, elle fait barrière et écran : associćc à un système de concours - expression du principe méritocratique - elle forme une pièce maîtresse dans la défense des particularismes des corps et des administrations, une protection contre le patronage politique et la concurrence du personnel parlementaire. Elle constitue très précisement une technologie d'autonomisation.

En même temps, on saisit comment, l'Ecole, incarnant institutionncllement la science politique, déplace le centre de gravité du champ de production idélogique et, corrélativement, modifie le poids relatif du champ politique, précisément par la concurrence avec le personnel politique qu'elle ouvre sur le marché des biens politiques dans sa lutte pour la définition du pensable et du possible politiques, mais aussi par la redéfinition des ressources politiques qu'elle produit.

Certes, on se demandera ce qui aujourd'hui demeure des savoirs de l'Ecole. Mais pense-t-on pour autant en avoir fini avec les usages politiques de la science. Sans même parler des avatars divers de l'historicisme, et pour prendre des exemples indigènes, on rappellera les aventures de la planification ou de la comptabilité nationale. Dans le champ de production idéologique, la tension est toujours vive entre science et politique, et permanent l'appel ou l'appropriation de la science par la politique. Pense-ton encore en avoir terminé avec la concurrence entre les professionnels de la politique et ces amateurs de politique, par métier ou par "vocation", que rassemble la catégorie d'intellectuels. En fait, l'Ecole a contribué à structurer la lutte entre trois types de spécialistes et de producteurs politiques, le personnel politique, les hauts-fonctionnaires et les intellectuels politistes. Ainsi, elle annonce, en l'engendrant, l'avenir. Coopération et confrontation n'ont pas cessé entre agents du champ politique et généralistes pourvus de la légitimité de l'expertise technique ou de la compétence savante, et si la configuration de cette lutte s'est modifiée, variant en fonction des tendances et des conjonctures politiques et sociales, elle n'a pas disparu. Dans les conflits de frontière entre le politique et le technique, dans l'opposition des ressources qui distinguent experts et personnel politique, dans la compétition pour la production et la sélection des biens politiques, l'Ecole libre des Sciences Politiques fait signe à l'E.N.A. et perdure.

Dominique Damamme Professeur à l'Université Montpellier 1 